\title{
Design of Normal-conducting Quadrupole Magnets for Linac4 at CERN
}

\author{
Liesbeth Vanherpe, Olivier Crettiez, Alexey Vorozhtsov, and Thomas Zickler
}

\begin{abstract}
Linac4 is the first element of the LHC Injectors Upgrade Project and will replace the existing Linac2 as linear injector of protons for the CERN accelerators. A new transfer line will link Linac4 to the Proton Synchrotron Booster (PSB). Approximately hundred normal-conducting electro-magnets are required for beam steering and focusing along the linac and the transfer line. This text concentrates on the design of the Linac4 quadrupole magnets. The design and the first magnetic measurements of the inter-tank quadrupole electro-magnets are discussed. In addition, the design and optimization of the transfer line quadrupole magnets are highlighted. The compatibility of the magnetic requirements and the power converter constraints for the latter magnet type is assessed.
\end{abstract}

Index Terms-Accelerator magnets, Linac4, Normalconducting magnets, Iron-dominated magnets, Design methodology

\section{INTRODUCTION}

$\mathbf{L}$ INAC4 is the first element of the upgrade project that aims to strengthen the performance of the Large Hadron Collider (LHC) at CERN, by progressively replacing or upgrading the accelerators presently used as injectors [1]-[3]. The new Linac4 is a normal-conducting linear accelerator for negative hydrogen ions, accelerating particles to $160 \mathrm{MeV}$. A new transfer line will link Linac4 to the Proton Synchrotron Booster (PSB), into which the beam is injected after conversion of the negative hydrogen ions into positive hydrogen ions in an electron-stripping foil [4]. Linac4 will thus replace the current linear injector of protons for the CERN accelerators, namely Linac2, which delivers protons at $50 \mathrm{MeV}$.

In total, approximately hundred normal-conducting electromagnets are required for Linac4 [5]. Two types of quadrupole electro-magnets provide beam focusing between the various Linac4 accelerator tanks and in the Linac4 transfer line: the so-called inter-tank quadrupole electro-magnets and the transfer line quadrupole electro-magnets. Both magnets are operated in a pulsed mode, which minimizes r.m.s. power consumption and allows the use of air-cooled coils. In this way, manufacturing, installation, and operation costs are cut.

In this text, the two quadrupole magnet types are discussed. The design and first measurement results of the inter-tank quadrupole magnets are presented in Section II. The design

Manuscript received July 15, 2013

L. Vanherpe (phone: +41 22766 2880; e-mail: liesbeth.vanherpe@cern.ch), O. Crettiez (e-mail: olivier.crettiez@cern.ch), and T. Zickler (e-mail: thomas.zickler@cern.ch) are with European Laboratory for Particle Physics (CERN), CH-1211 Geneva 23, Switzerland

A. Vorozhtsov was with JINR, Dubna, Russia. He is now with European Laboratory for Particle Physics (CERN), CH-1211 Geneva 23, Switzerland (e-mail: alexey.vorozhtsov@cern.ch) of the transfer line quadrupole magnets is elaborated in Section III. A summary and conclusions are given in Section IV.

\section{INTER-TANK QUADRUPOLE ELECTRO-MAGNETS}

\section{A. Design Requirements and Constraints}

The design requirements and constraints for the inter-tank quadrupole electro-magnets, hereafter called inter-tank magnets, are listed in Table I. The limited space available in the Linac4 inter-tank regions imposes tight restrictions both on the longitudinal and the transversal dimensions of the final assembly of the inter-tank magnets. The magnetic field requirements are dictated by the beam dynamics requirements [6], [7]. The field quality is assessed through harmonic analysis, which is obtained by Fourier transform of the integral of the radial component $B_{r}$ of the magnetic flux density along the magnet axis on a cylinder defined by the good field region radius $r_{0}$ (see equation (1)) [8]. In the case of a quadrupole magnet, the normalized multipole components are the skew components $a_{n}=A_{n} / A_{2}$ and the normal components $b_{n}=B_{n} / B_{2}$. Finally, the magnet design has to be compatible with the characteristics of the foreseen power supply [9], taking into account the Linac4 operation mode.

$$
\begin{gathered}
A_{n}=\frac{2}{N} \sum_{k=0}^{N-1}\left(\int B_{r}\left(\phi_{k}, z\right) d z \cdot \cos \left(n \phi_{k}\right)\right), \\
B_{n}=\frac{2}{N} \sum_{k=0}^{N-1}\left(\int B_{r}\left(\phi_{k}, z\right) d z \cdot \sin \left(n \phi_{k}\right)\right), \\
\text { with } \phi_{k}=\frac{2 \pi}{N} \cdot k \text { and } n=1 \ldots N
\end{gathered}
$$

TABLE I

REQUIREMENTS AND CONSTRAINTS FOR THE INTER-TANK QUADRUPOLE ELECTRO-MAGNETS

\begin{tabular}{lcc}
\hline \hline Parameter & Value & Unit \\
\hline Aperture radius $r$ & 27 & $\mathrm{~mm}$ \\
Overall magnet length & $<105$ & $\mathrm{~mm}$ \\
Overall magnet radius & $<125$ & $\mathrm{~mm}$ \\
Good field region radius $r_{0}$ & 18 & $\mathrm{~mm}$ \\
Integrated magnetic flux density gradient & $1.83-2.22$ & $\mathrm{~T}$ \\
Harmonic content $b_{n}$ at $r_{0}$ & $<0.01(=100 \mathrm{units})$ \\
$\quad$ for $n=3,4, \ldots, 10$ & 100 & \\
Maximum power converter current & 900 & $\mathrm{~A}$ \\
Maximum voltage on power converter & Pulsed & \\
Operation mode & 2.0 & $\mathrm{~Hz}$ \\
Repetition frequency & 500 & $\mathrm{~ms}$ \\
Total duty cycle & & \\
\hline \hline
\end{tabular}




\section{B. Magnet Design}

Fig. 1 shows the final, basic magnet assembly of the intertank magnet. Detailed information on the magnet design can be found in [10]. First, the pole profile has been designed with two-dimensional calculations. Subsequently, with threedimensional simulations, a $45^{\circ}$ chamfer with a height of $4 \mathrm{~mm}$ has been applied to the poles at the yoke ends to further optimize the magnetic field quality. The height of the chamfer has been chosen based on the value of the resulting $b_{6}$ component. The field quality of the final magnet design is displayed in Fig. 2.

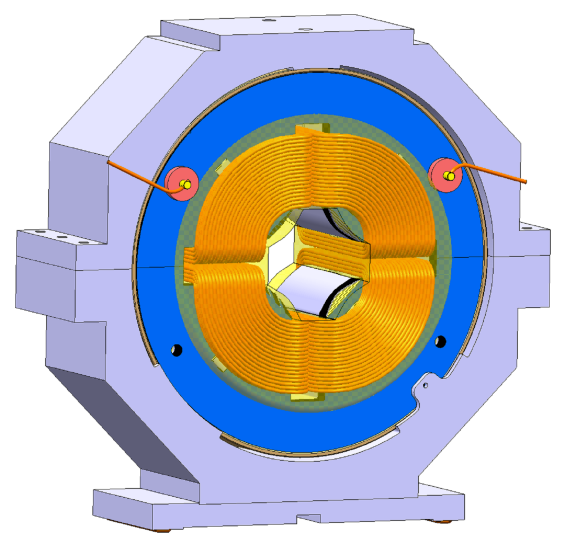

Fig. 1. Final assembly of the Linac4 inter-tank quadrupole electro-magnet.

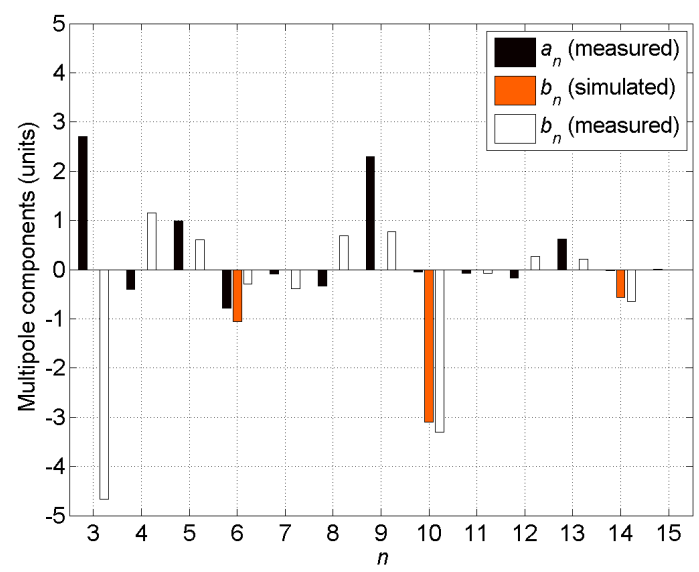

Fig. 2. Simulated and measured multipole components of the magnetic field of the inter-tank magnet [10], [11]. Measurements have been performed at $18 \%$ of the nominal current.

The yoke parts are produced by electrical discharge machining. The magnet is assembled from two yoke halves and four coils, held together by a shrink-fitted stainless steel ring. This structure reduces potential assembly errors, which can result in unwanted harmonic content. After assembly, the magnet is vacuum-impregnated with epoxy resin. Finally, a magnet holder made from anodized aluminium is installed around the magnet. The intertank magnets will be installed on a support with a keyway, and aligned with respect to the axis and the reference plane of the applicable Linac4 tank.

\section{Prototype Measurements}

The manufacturing has been started with the production of a prototype magnet, which has been received and tested at CERN [11]. The magnetic measurements have been performed with rotating coils, at $18 \%$ of the nominal current, which is compatible with the air-cooled coils powered for very short periods. Furthermore, the results have been averaged over measurements at opposite current levels in order to eliminate the offset due to remanent field, which tends to dominate the results at very low current, and capture only the component proportional to excitation current.

The measured magnetic field quality is shown in Fig. 2, which compares the measured multipole components with the calculated components. It can be seen that the measured harmonic content is much less then the maximum allowed content of 100 units (see Table I). Furthermore, the simulated allowed normal components $b_{10}$ and $b_{14}$ correspond very well to the measured values. The non-negligible $a_{3}$ and $b_{3}$ components indicate mechanical assembly errors and will be the subject of further measurement study on the series magnets. In addition, the effect of eddy currents has been tested, and a stabilization time of $1 \mathrm{~ms}$ was obtained. A detailed discussion of the measurement campaign for the inter-tank magnets can be found in [11].

\section{Status}

At the moment of writing, all inter-tank magnets have been manufactured. They will be tested both electrically and magnetically at CERN.

\section{TRANSFER LINE QUADRUPOLE ELECTRO-MAGNETS}

\section{A. Design Requirements and Constraints}

The design of the transfer line quadrupole electro-magnets, hereafter called transfer line magnets, has to satisfy the requirements and constraints listed in Table II [7], [12]. In addition, the electrical characteristics of the magnet should fall within the operation range of the foreseen MaxiDiscap power converter [9], [13], which is illustrated in Fig. 3.

TABLE II

REQUIREMENTS AND CONSTRAINTS FOR THE TRANSFER LINE QUADRUPOLE ELECTRO-MAGNETS

\begin{tabular}{lcc}
\hline \hline Parameter & Value & Unit \\
\hline Aperture radius $r$ & 50 & $\mathrm{~mm}$ \\
Good field region radius $r_{0}$ & 37.5 & $\mathrm{~mm}$ \\
Magnetic length $l_{\mathrm{m}}$ & 300 & $\mathrm{~mm}$ \\
Magnetic flux density gradient & 6 & $\mathrm{~T} / \mathrm{m}$ \\
Integrated magnetic flux density gradient & 1.8 & $\mathrm{~T}$ \\
Harmonic content $b_{n}$ at $r_{0}$ & $<0.01$ & \\
$\quad$ for $n=3,4, \ldots, 10$ & & \\
Maximum voltage on power converter & 1 & $\mathrm{kV}$ \\
Operation mode & pulsed & \\
Repetition frequency & 1.111 & $\mathrm{~Hz}$ \\
Total duty cycle & 900 & $\mathrm{~ms}$ \\
\hline \hline
\end{tabular}




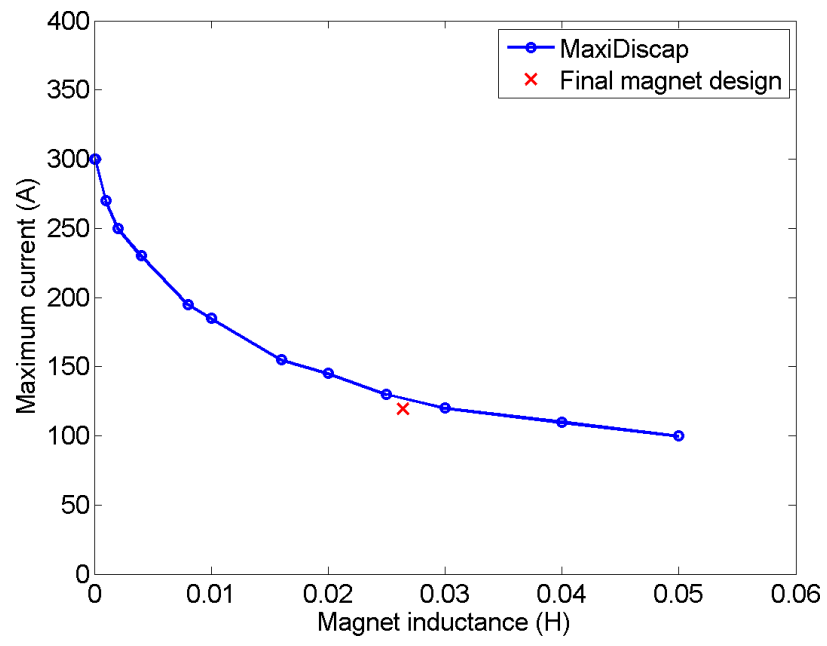

Fig. 3. MaxiDiscap power converter operation range: delivered current as a function of the inductance load [13].

\section{B. Assessment of Requirements and Constraints}

For a magnet with non-saturated iron yoke, the stored magnetic energy $E_{s}$ relates to the inductance and the current as [14], [15]:

$$
E_{\mathrm{s}}=\frac{L I^{2}}{2} .
$$

The inductance defined by equation (2) is the so-called energyequivalent inductance $L$ of a magnet [8], [14], [16]. The inductance $L$ can be calculated based on the value of $E_{\mathrm{s}}$ obtained from simulations, where the magnetic field is known throughout the magnet model and is coupled with the excitation current $I$. Alternatively, for an iron-dominated quadrupole electro-magnet with racetrack coils, the stored energy can be estimated as

$$
E_{\mathrm{s}}=\pi \frac{1}{\mu_{0} \eta^{2}}\left(\int_{-\infty}^{+\infty} G(0,0, z) \mathrm{d} z\right)^{2} l_{\mathrm{m}}^{-1} r^{4},
$$

with $\mu_{0}$ the permeability in free space, a design efficiency of $\eta=95 \%, G$ the magnetic flux density gradient, $l_{\mathrm{m}}$ the magnetic length, which is defined as

$$
l_{\mathrm{m}}=\frac{\int G(0,0, z) \mathrm{d} z}{G(0,0,0)},
$$

and $r$ the magnet aperture radius [15].

Equation (3) is a semi-analytical equation, which can be used for fast parametric analysis: for a given integrated gradient, it can be verified which values of the parameters $l_{\mathrm{m}}$ and $r$ can result in a magnet design that is compatible with a given power converter. Fig. 4 displays the result of this analysis for the integrated gradient required for the transfer line magnet. Feasible combinations of $l_{\mathrm{m}}$ and $r$ are indicated with green circles, unfeasible combinations with red crosses. It can be seen that the magnetic requirements of Table II are compatible with the power converter. In [17], it is shown how this has been valuable in defining the final design requirements for the transfer line magnet.

For the transfer line magnet, the stored magnetic energy as estimated with equation (3) is $187 \mathrm{~J}$. With equation (2), it

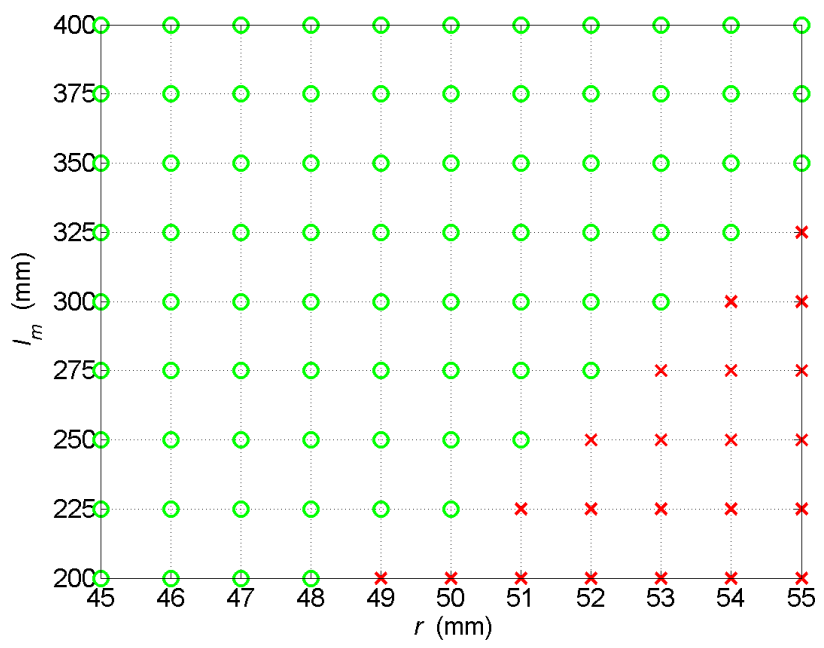

Fig. 4. Magnetic design for an integrated gradient of $1.8 \mathrm{~T}$ with the MaxiDiscap power converter: feasible combinations of $l_{\mathrm{m}}$ and $r$ are indicated with green circles, unfeasible combinations with red crosses.

can be verified that several corresponding $(L, I)$-couples are indeed within the operation range of the MaxiDiscap power converter.

\section{Magnet Design}

First, the pole profile has been designed with twodimensional calculations. Second, during three-dimensional simulations, end chamfers have been applied to the four poles, as illustrated in Fig. 5. The height of these $45^{\circ}$ chamfers been chosen equal to $8.5 \mathrm{~mm}$, based on the value of the $b_{6}$ component of the field obtained for different chamfer heights, which is displayed in Fig. 6. The final chamfer height will be confirmed after magnetic measurements.

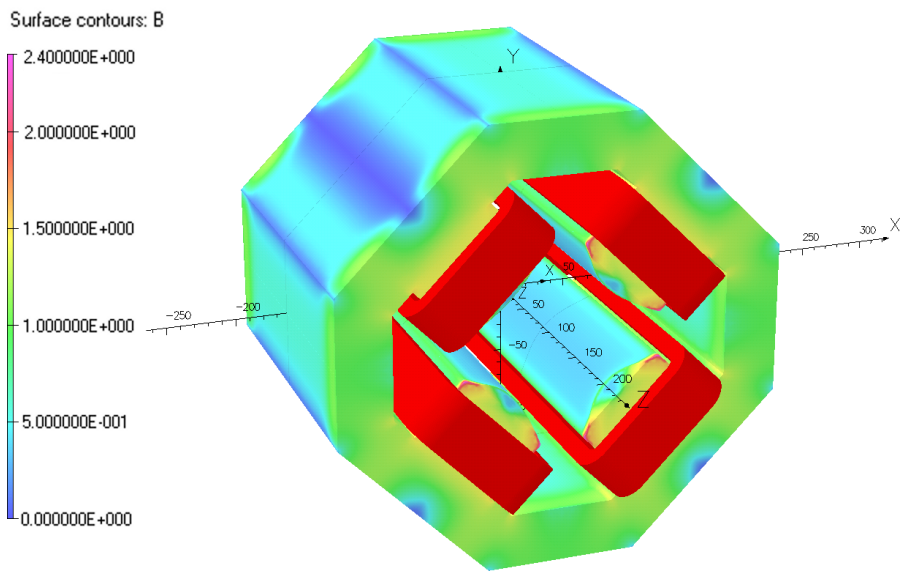

Fig. 5. Transfer line magnet with a map of the magnetic flux density on the yoke surface.

Fig. 3 confirms that the electrical characteristics of the final magnet design are compatible with the operation range of the power converter. More details on the design of the transfer line magnet can be found in [17]. 


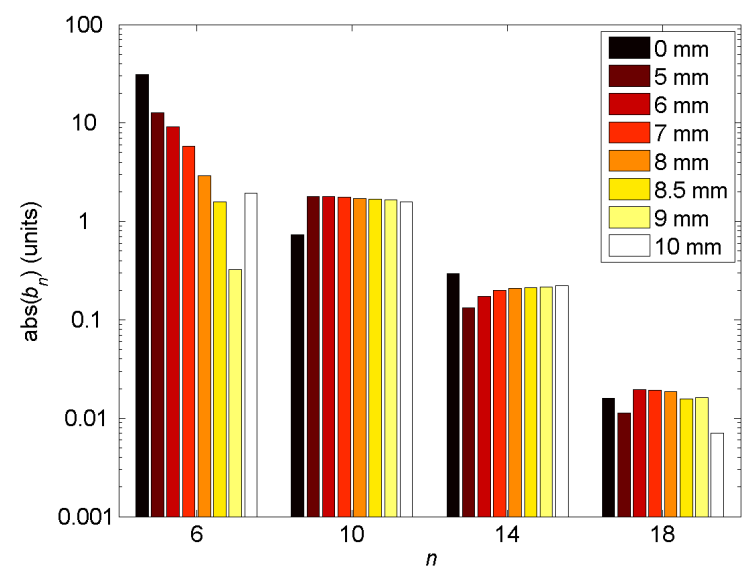

Fig. 6. Harmonic analysis for different values of the chamfer height.

\section{Effect of the Vacuum Chamber}

In the final transfer line magnet assembly, a vacuum chamber will be installed in the magnet aperture. In order to estimate the effect of eddy currents in the vacuum chamber wall on the magnetic field, two-dimensional simulations have been performed for the parameter values listed in Table III. Fig. 7 illustrates the evolution of the magnetic flux density $B_{\text {mod }}$ at $(x, y)=(33.6,33.6)$, which is inside the vacuum chamber and close to the pole tip. The deviation from the ideal ramp-up is caused by eddy currents in the vacuum chamber wall.

TABLE III

PARAMETER VALUES OF EDDY CURRENTS SIMULATION

\begin{tabular}{lcc}
\hline \hline Parameter & Value & Unit \\
\hline Wall thickness & 1.5 & $\mathrm{~mm}$ \\
Insulation gap & 1 & $\mathrm{~mm}$ \\
Material & SS $316 \mathrm{LN}$ & \\
Conductivity & $1.33 \times 10^{3}$ & $\mathrm{~S} / \mathrm{mm}$ \\
Relative permeability & 1.001 & \\
\hline Cycle type & ramp-up & \\
Maximum current & 119.6 & $\mathrm{~A}$ \\
Rise time & 5 & $\mathrm{~ms}$ \\
Full cycle time & 1 & $\mathrm{~s}$ \\
\hline \hline
\end{tabular}

The stabilisation time $t_{v c}$ is defined as the time interval that elapses between the end of the ramp-up at $t=5 \mathrm{~ms}$, and the point in time when the magnetic flux density comes within a distance $\epsilon$ of its stable value. For $\epsilon=1 \times 10^{-4}$, the value $t_{v c}=0.20 \mathrm{~ms}$ is obtained.

\section{E. Status}

At the time of writing, a prototype magnet has been manufactured, but no magnetic measurements have been performed. The planned measurements are similar to those of the intertank magnet (see Section II-C).

\section{CONCLUSION}

In this text, first, the design of the Linac4 inter-tank quadrupole electro-magnets has been presented [10]. The
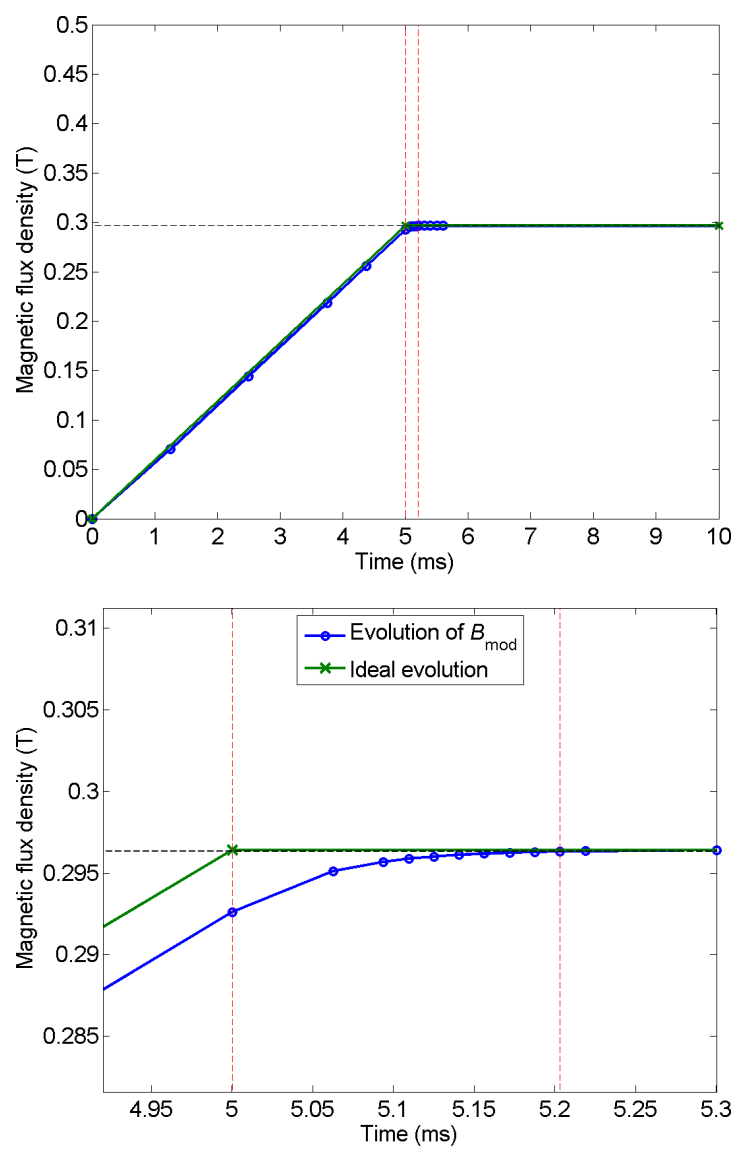

Fig. 7. Evolution of $B_{\bmod }$ at $(x, y)=(33.6,33.6)$, close to the pole tip, inside the vacuum chamber.

results of the magnetic measurements of the prototype magnet confirm that the magnet design satisfies the magnetic field requirements [11]. Next, the design of the transfer line quadrupole electro-magnets has been discussed. In particular, the compatibility of the design requirements and constraints has been assessed, based on the work of [15], which allows to check whether a given set of magnetic field requirements is compatible with a given pulsed power converter, before the start of magnetic field simulations. Furthermore, twodimensional simulations have been performed to estimate the effect of eddy currents in the vacuum chamber wall on the magnetic field.

\section{ACKNOWLEDGEMENTS}

The authors gratefully acknowledge Regis Chritin, Peter Galbraith, and Marco Buzio for the execution and the processing of the magnetic measurements on the Linac4 inter-tank quadrupole electro-magnet prototype.

\section{REFERENCES}

[1] L. Arnaudon, P. Baudrenghien, M. Baylac, G. Bellodi, Y. Body, J. Borburgh, P. Bourquin, J. Broere, O. Brunner, L. Bruno, C. Carli, F. Caspers, S. M. Cousineau, Y. Cuvet, C. De Almeida Martins, T. Dobers, T. Fowler, R. Garoby, F. Gerigk, B. Goddard, K. Hanke, M. Hori, M. Jones, K. Kahle, W. Kalbreier, T. Kroyer, D. Küchler, A. M. Lombardi, L. A. López-Hernandez, M. Magistris, M. Martini, S. Maury, E. Page, M. Paoluzzi, M. Pasini, U. Raich, C. Rossi, 
J. P. Royer, E. Sargsyan, J. Serrano, R. Scrivens, M. Silari, M. Timmins, W. Venturini-Delsolaro, M. Vretenar, R. Wegner, W. Weterings, and T. Zickler, "Linac4 Technical Design Report," CERN, Geneva, Tech. Rep. CERN-AB-2006-084, CARE-Note-2006-022-HIPPI, December 2006.

[2] M. Vretenar, "Status of Linac4 construction at CERN," CERN, Geneva, Tech. Rep. CERN-ATS-2010-227, September 2010.

[3] L. Arnaudon, P. Baudrenghien, C. Bertone, Y. Body, J. Broere, O. Brunner, M. Buzio, C. Carli, F. Caspers, J. Corso, J. Coupard, A. Dallocchio, N. Dos Santos, R. Garoby, F. Gerigk, L. Hammouti, K. Hanke, M. Jones, I. Kozsar, J. Lettry, J. Lallement, A. Lombardi, L. López-Hernandez, C. Maglioni, S. Mathot, S. Maury, B. Mikulec, D. Nisbet, C. Noels, M. Paoluzzi, B. Puccio, U. Raich, S. Ramberger, C. Rossi, N. Schwerg, R. Scrivens, G. Vandoni, S. Weisz, J. Vollaire, M. Vretenar, and T. Zickler, "The LINAC4 project at CERN," CERN, Geneva, Tech. Rep. CERN-ATS-2011-041, August 2011.

[4] B. Goddard, M. Aiba, C. Bracco, C. Carli, M. Meddahi, and W. J. M. Weterings, "Stripping foil issues for h- injection into the cern psb at 160 mev," in Proceedings of IPAC10, June 2010, p. 4 p.

[5] F. Borgnolutti, O. Crettiez, A. Newborough, L. Vanherpe, A. Vorozhtsov, and T. Zickler, "Status of the new Linac4 magnets at CERN," in Proceedings of IPAC11, 2011.

[6] M. Garcia Tudela, G. Bellodi, M. Eshraqi, L. M. Hein, J. B. Lallement, A. Lombardi, P. A. Posocco, and E. Sargsyan, "Updated end-to-end simulations of Linac4," CERN, Geneva, Tech. Rep. CERN-sLHCProject-Note-0025, November 2010.

[7] G. Bellodi, M. Eshraqi, M. G. Tudela, L. Hein, J.-B. Lallement, S. Lanzone, A. Lombardi, P. Posocco, and E. Sargsyan, "Alignment and field error tolerance in Linac4," CERN, Tech. Rep. CERN-ATS-Note2011-021, April 2011.

[8] S. Russenschuck, Field Computation for Accelerator Magnets. WileyVCH, Berlin, 2010.

[9] C. de Almeida Martins, G. Le Godec, M. Cerquieira Bastos, and Q. King, "Work package description: power converters and klystron modulators," EDMS 976137, January 2009.

[10] A. Vorozhtsov, "Linac4 Inter-tank Electromagnetic Quadrupole," CERN, Tech. Rep. EDMS 1183024, 2012.

[11] M. Buzio, R. Chritin, and P. Galbraith, "Magnetic measurements of Linac4 intertank electromagnetic quadrupole prototype," CERN, Tech. Rep. EDMS 1265033, 2013.

[12] C. Carli, M. Garcia Tudela, A. Lombardi, L. Hein, and M. Eshraqi. (2011, September) L4 transfer line to the PSB - update from Oct 2010. CERN. [Online]. Available: http://indico.cern.ch/getFile. py/access? contribId=4\&resId=1\&materialId=slides \& confId=149883

[13] J.-M. Cravero, "MaxiDiscap operation range," Private communication, 2010, CERN.

[14] D. Tommasini, "Practical definitions and formulae for magnets," CERN, Tech. Rep. EDMS 1162401, 2011.

[15] L. Vanherpe and T. Zickler, "A predictive software tool for compatibility assessment of magnet design requirements and power converter constraints based on the stored magnetic energy," IEEE Trans. Magn., to be published.

[16] M. Buzio, R. Chritin, and D. Giloteaux, "Measurement of the inductance of resistive magnets: two case studies," CERN, Tech. Rep. CERN-ATSNote-2011-047 TECH, EDMS 1148084, 2011.

[17] L. Vanherpe, "Design report of the Linac4 Transfer Line Quadrupole Electromagnets,” CERN, Tech. Rep. EDMS 1291572, 2013. 